\title{
Maximizing expected utility over a knapsack constraint
}

\author{
Jiajin $\mathrm{Yu}^{\mathrm{a}}$, Shabbir Ahmed ${ }^{\mathrm{b}, *}$ \\ ${ }^{a}$ College of Computing, Georgia Institute of Technology, Atlanta, GA 30332 \\ ${ }^{b}$ School of Industrial \& Systems Engineering, Georgia Institute of Technology, Atlanta, GA 30332
}

\begin{abstract}
The expected utility knapsack problem is to pick a set of items with random values so as to maximize the expected utility of the total value of the items picked subject to a knapsack constraint. We devise an approximation algorithm for this problem by combining sample average approximation and greedy submodular maximization. Our main result is an algorithm that maximizes an increasing submodular function over a knapsack constraint with an approximation ratio better than the well known (1-1/e) factor.
\end{abstract}

Keywords: Knapsack problem, Utility maximization, Submodularity, Sample Average Approximation, Approximation algorithm

2000 MSC: 90C15 68W25

\section{Introduction}

This paper develops an approximation algorithm for the expected utility knapsack problem. Given a ground set of $n$ items $\mathcal{U}=\{1, \ldots, n\}$; a random non-negative vector of values $\tilde{a}$ for the items; a positive integer vector $b$ of weights for the items; a positive integer capacity of $B$; and a utility function $f: \mathbb{R}_{+} \mapsto \mathbb{R}_{+}$; the expected utility knapsack problem is to pick a subset $S$ of items so to

$$
\max _{S \subseteq \mathcal{U}}\{F(S):=\mathrm{E}[f(\tilde{a}(S))] \mid b(S) \leq B\}
$$

where $x(S):=\sum_{i \in S} x_{i}$. Note that the expectation above is with respect to the distribution of $\tilde{a}$. Throughout the paper, we assume $f(0)=0$ and $f(a(S)) \geq 1$ for any $a \sim$ $\tilde{a}$ and $S \neq \emptyset$. Therefore $F(\emptyset)=0$ and $F(S) \geq 1$ for $S \neq \emptyset$. We further assume that the utility function $f$ is strictly increasing and concave which correspond to riskaverse preferences 1, 2. Commonly used utility functions such as $\log$-utility $f(t)=\log t$, exponential utility $f(t)=$ $1-e^{-\alpha t}$ for $\alpha>0$, and power utility $f(t)=t^{p}$ for $0<p<$ 1 , all satisfy this assumption.

Concavity of $f$ along with the non-negativity of $\tilde{a}$ imply that the expected utility $F$ is a submodular function of the selected set $S$ (cf. [3]). Accordingly, (SP) is a submodular maximization problem with a knapsack constraint. It is well known that in general the approximation ratio for such problems is bounded by $(1-1 / e)$ 4. Moreover a variant of the greedy algorithm achieves this bound [5]. However these results assume a value oracle model where

\footnotetext{
* Corresponding author

Email addresses: jiajinyu@gatech.edu (Jiajin $\mathrm{Yu}$ ), sahmed@isye.gatech.edu (Shabbir Ahmed)
}

the underlying submodular function is general and can be evaluated exactly.

In $(\mathrm{SP})$ evaluation of $F$ requires evaluating a multidimensional integral over the distribution of $\tilde{a}$. Moreover, the distribution of $\tilde{a}$ may not be explicitly available, but only available through a sampling oracle. In such a setting, exact evaluation of $F$ is impossible. In this paper we adopt the sample average approximation (SAA) framework [6] towards approximately evaluating $F$. In SAA the original distribution of the uncertain parameters is replaced by an empirical distribution by sampling a certain number of scenarios.

The sample average approximation of $(\mathrm{SP})$ is

$$
\max _{S \subseteq \mathcal{U}}\left\{F^{N}(S)=\frac{1}{N} \sum_{i=1}^{N} f\left(a_{i}(S)\right) \mid b(S) \leq B\right\}
$$

where $\left\{a_{1}, \ldots, a_{N}\right\}$ is an i.i.d sample of $\tilde{a}$. Note that $F^{N}$ is a submodular function and $\mathrm{SA}$ ) is a deterministic knapsack constrained submodular maximization problem. It follows from classical SAA theory [6] that by solving (SA) corresponding to a sufficient number of samples $N$ using an approximation algorithm of a given absolute error $\delta$, with high probability, we can obtain a solution to the original problem (SP whose absolute error is not too large compared to $\delta$. Moreover the required sample size $N$ is polynomial with respect to problem dimension.

If $\mathrm{SA}$ is solved using a relative error approximation algorithm (such as those in the submodular optimization literature) we need to adapt the SAA theory to recover a corresponding relative error for the true problem $(\mathrm{SP})$. We make this adaptation. Further we develop an approximation algorithm for solving (SA) based on maximizing increasing submodular functions over a knapsack constraint. Specifically, the contribution of this paper is two-fold: 
SAA analysis under relative error: We prove that with high probability only a polynomial number of samples is enough for an approximation algorithm that solves the SAA problem with relative error to give an approximate solution to the true stochastic problem of similar relative error. The works by Shmoys and Swamy [7], and Charikar et al. 8] are most relevant to our work as they both considered approximation algorithms with relative error for 2-stage stochastic optimization, rather than the absolute error usually considered in stochastic programming. However the polynomial sample size in their results depends on a ratio between the cost of the first stage and the cost of the second stage, which is not applicable to our single stage setting. A result similar to ours is mentioned in [9, pp. 21] but without detailed proof.

Increasing submodular maximization over a knapsack: The increasing and concavity properties of common utility functions imply that $\mathrm{SA}$ involves maximizing an increasing submodular function over a knapsack constraint. Sviridenko [5] recently developed a greedy algorithm to maximize an increasing submodular function over a knapsack constraint with approximation ratio $(1-1 / e)$. We adapt this algorithm and its analysis exploiting the strict monotonicity of the utility function and show an approximation ratio better than the $(1-1 / e)$ bound. For power utility functions, we explicitly characterize the approximation ratio as a function of the budget $B$ and the exponent of the power function. Some other works that have improved on the $(1-1 / e)$ bound are by Conforti and Cornuéjols [10, and Vondràk [11. However these consider cardinality constraints and matroid constraints, respectively, and are not applicable in our knapsack setting.

We close this section with a brief discussion of some additional related literature. $\mathrm{Li}$ and Deshpande 12 study the problem of maximizing expected utility for various combinatorial optimization problems. They assume that the random coefficients are independent to simplify the expectation operation and use an approximation of the utility function. We allow more general distribution but are restricted to the knapsack setting. Klastorin [13 study a similar problem but he assumes exact evaluation of the expectation objective and gives an algorithm that solves a continuous relaxation of the problem and then uses that in a branch-and-bound algorithm. Asadpour et.al. 14] study maximizing a stochastic submodular function under matroid constraints. They assume exact evaluation of the expectation objective and do not consider increasing submodular functions. Mehrez and Sinuany-Stern [15] study a variation of the problem arising in resource allocation applications, but in their model the utility of items are separable which is different from our setting. Dean et.al [16] studied a variation where sizes of the elements are independent random variables while the values of the elements are deterministic. They devised both "nonadaptive" and "adaptive" policies to choose items in order to maximize the expected value of items that can be fit in the knapsack.

\section{Sample Average Approximation}

In this section, we adapt the classical SAA theory (cf. 17]) which corresponds to an absolute error setting to our required setting of relative error. We consider a generalization of $\mathrm{SP})$ :

$$
\max _{S \subseteq \mathcal{U}}\{F(S)=\mathrm{E}[f(\tilde{a}, S)] \mid S \in X\},
$$

where $X$ is the constraint set (e.g. knapsack constraint) and $f: 2^{\mathcal{U}} \mapsto \mathbb{R}_{+}$, parameterized by $a$, is a nonnegative set function. The sample average approximation of (SP0) is

$$
\max _{S \subseteq \mathcal{U}}\left\{F^{N}(S)=\frac{1}{N} \sum_{i=1}^{N} f\left(a_{i}, S\right) \mid S \in X\right\},
$$

where $\left\{a_{1}, \ldots, a_{N}\right\}$ is an i.i.d sample of $\tilde{a}$. Then $\sqrt{\mathrm{SP}}$ is a special case of (SP0) and (SA) is a special case of (SA0). Let $S^{*}$ be an optimal solution of (SP0). We make the following assumption on $f(a, S)$ and $\mathrm{E}[f(\tilde{a}, S)]$.

Assumption 1. For any $a \sim \tilde{a}, S \in X$, and $S \neq \emptyset$, we assume $f(a, S) \geq 1$. Therefore $F^{N}(S) \geq 1$ and $F(S) \geq 1$. For any $S \in X$, we also assume $\mathrm{E}[f(\tilde{a}, S)]$ is well-defined and finite, and $\mathrm{E}\left[e^{t f(\tilde{a}, S)}\right]$ is finite in a neighborhood of $t=0$.

Using the above assumption and standard Large Deviation analysis (cf. [6]), we can show that if $N$ is large enough, for every $S \in X, F^{N}(S)$ is close to $F(S)$ in a relative sense.

Lemma 2. Given $\gamma>0$, let $\sigma^{2}=$ $\max \{\operatorname{Var}[f(\tilde{a}, S)] \mid S \in X\} \quad$ and $S^{*}$ be an optimal solution of the problem. If $N \geq \frac{2 \sigma^{2}}{\epsilon^{2}} \log \frac{|X|}{\gamma}$, then

$$
\operatorname{Pr}\left\{\bigcap_{S \in X}\left|F(S)-F^{N}(S)\right| \leq \epsilon F\left(S^{*}\right)\right\} \geq 1-2 \gamma .
$$

Proof. Let $\left\{a_{1}, \ldots, a_{N}\right\}$ be the i.i.d sample defining $F^{N}(S)$. Let $A_{1}$ be the event that there exists a set $S$ such that $F(S)-F^{N}(S)>\epsilon F\left(S^{*}\right)$, and let $A_{2}$ be the event that there exists a set $S$ such that $F^{N}(S)-F(S)>\epsilon F\left(S^{*}\right)$. Let $\sigma_{S}^{2}=\operatorname{Var}[f(\tilde{a}, S)]$, then $\sigma^{2}=\max _{S \in X} \sigma_{S}^{2}$. If we can show that when $N \geq \frac{2 \sigma^{2}}{\epsilon^{2}} \log \frac{|X|}{\gamma}$ we have $\operatorname{Pr}\left\{A_{1}\right\} \leq \gamma$ and $\operatorname{Pr}\left\{A_{2}\right\} \leq \gamma$ then we have the desired inequality (1).

Let us prove that $\operatorname{Pr}\left\{A_{1}\right\} \leq \gamma$.

$$
\begin{aligned}
& \operatorname{Pr}\left\{\bigcup_{S \in X} F(S)-F^{N}(S)>\epsilon F\left(S^{*}\right)\right\} \\
\leq & \sum_{S \in X} \operatorname{Pr}\left\{F(S)-F^{N}(S)>\epsilon F(S)\right\} \\
= & \sum_{S \in X} \operatorname{Pr}\left\{F^{N}(S)<(1-\epsilon) F(S)\right\}
\end{aligned}
$$

By Assumption 1, we know that $F(S)$ is finite for every $S$ and $\mathrm{E}\left[e^{t f(\tilde{a}, S)}\right]$ is finite in a neighborhood of $t=0$. So if 
$\epsilon$ is sufficiently small, by Large Deviation Theory (cf. [17. Sec 7.2.8]), we have

$$
\operatorname{Pr}\left\{F^{N}(S)<(1-\epsilon) F(S)\right\} \leq \exp \left(-\frac{N(\epsilon F(S))^{2}}{2 \sigma_{S}^{2}}\right) .
$$

Thus

$$
\begin{aligned}
\sum_{S \in X} \operatorname{Pr}\left\{F^{N}(S)<(1-\epsilon) F(S)\right\} & \leq \sum_{S \in X} \exp \left(-\frac{N(\epsilon F(S))^{2}}{2 \sigma_{S}^{2}}\right) \\
& \leq|X| \exp \left(-\frac{N \epsilon^{2}}{2 \sigma^{2}}\right) \\
& \leq \gamma
\end{aligned}
$$

which proves $\operatorname{Pr}\left\{A_{1}\right\} \leq \gamma$. The proof for $\operatorname{Pr}\left\{A_{2}\right\} \leq \gamma$ is identical, which we omit here.

Equipped with the lemma above, we are ready to show that we can use any algorithm that solves (SA0) approximately to solve (SP0) without losing too much.

Theorem 3. Given an algorithm that solves (SA0) with approximation ratio $\beta$, with probability at least $1-2 \gamma$, we can use the same algorithm to solve the stochastic problem (SP0) with approximation ratio $\beta(1-\epsilon)-\epsilon$ by sampling $\tilde{a}$ at least $\frac{2 \sigma^{2}}{\epsilon^{2}} \log \frac{|X|}{\gamma}$ times.

Proof. If we sample $N \geq \frac{2 \sigma^{2}}{\epsilon^{2}} \log \frac{|X|}{\gamma}$ times of $\tilde{a}$, by Lemma 2, we know that for any $S^{\prime} \in X$, we have $\left|F\left(S^{\prime}\right)-F^{N}\left(S^{\prime}\right)\right| \leq \epsilon F\left(S^{*}\right)$ with probability at least $1-2 \gamma$. Assume the event happens. Let $S^{*}$ be an optimal solution of $(\mathrm{SP} 0)$. Let $S$ be an $\beta$-approximation solution of SA0. Then $F^{N}(S) \geq \beta F^{N}\left(S^{*}\right)$. We have

$$
\begin{array}{rlr}
F(S) & \geq F^{N}(S)-\epsilon F\left(S^{*}\right) & \text { (by Lemma 2) } \\
& \geq \beta F^{N}\left(S^{*}\right)-\epsilon F\left(S^{*}\right) & \\
& \geq \beta\left(F\left(S^{*}\right)-\epsilon F\left(S^{*}\right)\right)-\epsilon F\left(S^{*}\right) \quad(\text { by Lemma 2) } \\
& =(\beta(1-\epsilon)-\epsilon) F\left(S^{*}\right) .
\end{array}
$$

Remark 4. Note that $\sigma^{2}$ is a problem specific parameter. We can bound $\sigma^{2}$ under additional assumptions (see Theorem 8 in Section 3.2.)

\section{Increasing Submodular Maximization over a Knapsack}

In this section, we will give an algorithm for maximizing a nonnegative increasing submodular function over a knapsack constraint. Recall the universe is $\mathcal{U}=\{1, \ldots, n\}$ and the weight of element $i$ is $b_{i} \in \mathbb{N}_{+}$. Let $F: 2^{\mathcal{U}} \mapsto \mathbb{R}_{+}$ be a nonnegative increasing function. It is a submodular function if and only if

$$
F(S \cup\{i\})-F(S) \geq F(T \cup\{i\})-F(T), \forall S \subseteq T, i \notin T .
$$

Another property of an increasing submodular function is the following:

$$
F(T) \leq F(S)+\sum_{i \in T \backslash S}(F(S \cup\{i\})-F(S)), \forall S, T \subseteq \mathcal{U} .
$$

The problem we are interested in is of the following general form:

$$
\max _{S \subseteq \mathcal{U}}\{F(S) \mid b(S) \leq B\} .
$$

Following Sviridenko [5], we propose Algorithm 1 to solve $(\mathrm{P}$ approximately. The algorithm first picks the best set $S_{1}$ among all sets of size less than a prescribed constant $K$. In the second step, for each set of size $K$, the algorithm greedily packs items into the set. Let the best set in this step be $S_{2}$. Finally, the algorithm outputs the better of $S_{1}$ and $S_{2}$. The main departure from the algorithm in [5] is that here we enumerate and extend all sets of size $K$, instead of sets of size 3 as in [5].

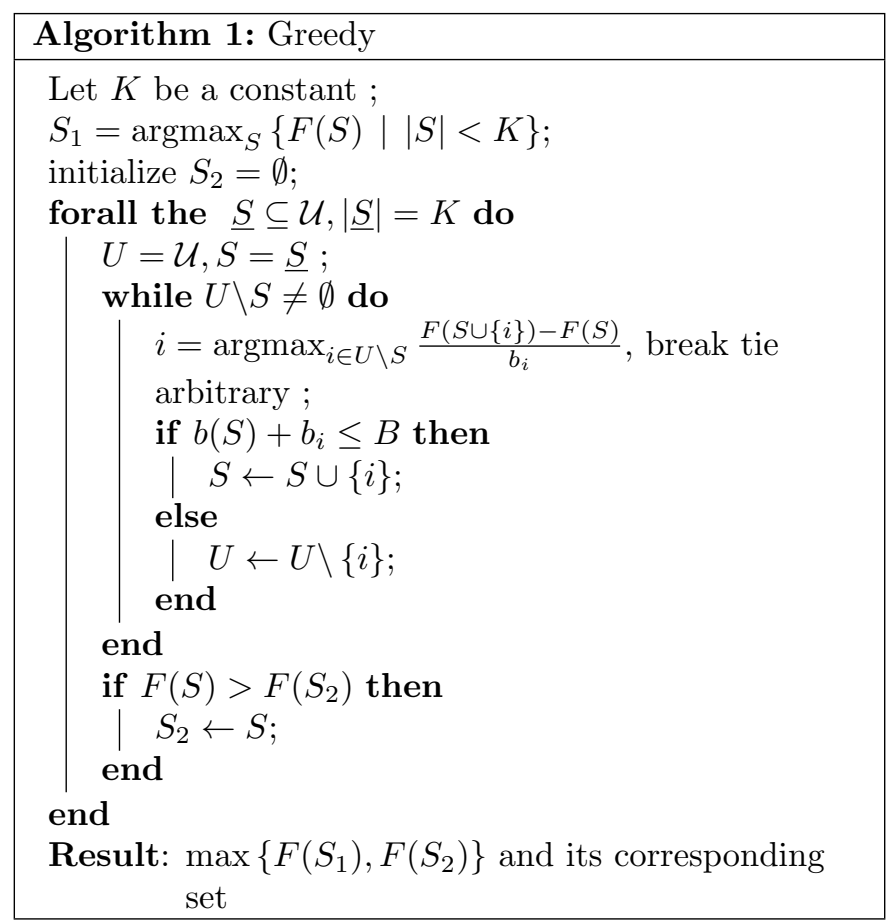

The constant $K$ used in Algorithm 1 is based on the following measure of monotonicity of the increasing function $F(S)$ :

Definition 5. Given an instance of $(\mathrm{P})$, let $S^{*}$ be an optimal solution of $(\mathrm{P})$. We define

$$
\alpha=\max _{i \notin S^{*}} \frac{F\left(S^{*} \cup\{i\}\right)-F(\{i\})}{F\left(S^{*}\right)-F(\{i\})} .
$$

Note that since $F$ is increasing, $\alpha>1$. In Section 3.1 we prove the following result.

Theorem 6. Given a nondecreasing submodular function $F$ with $\alpha$, by setting $K=\left\lceil e^{\alpha^{\prime}}\right\rceil$ for any $\alpha^{\prime} \geq 1$, Algorithm 
1 solves the problem with an approximation ratio of at least $1-e^{-\min \left(\alpha, \alpha^{\prime}\right)}$.

Remark 7. Note that since $F$ is increasing, even if we do not know the exact value of $\alpha$ as defined in (3), by setting $K=\left\lceil e^{\alpha^{\prime}}\right\rceil$ with $\alpha^{\prime}>1$, Algorithm 1 is a polynomial time algorithm with approximation ratio strictly better than the $(1-1 / e)$ ratio of the algorithm proposed in [5].

\subsection{Proof of Theorem 6}

Without loss of generality, we assume that any optimal solution of $(\mathrm{P})$ is of size at least $K$, because otherwise we would find an optimal solution as $S_{1}$. Let $S^{*}$ be an optimal set of size at least $K$. Before going further, we define a subset of $S^{*}$ of size $K$. We order the items in $S^{*}$ such that the $t$-th element $j_{t}$ is an $i$ achieving $\max \left(F\left(\left\{j_{1}, \ldots, j_{t-1}\right\} \cup\{i\}\right)-F\left(\left\{j_{1}, \ldots, j_{t-1}\right\}\right)\right)$ among $i \in S^{*} \backslash\left\{j_{1}, \ldots, j_{t-1}\right\}$. Let $Y=\left\{j_{1}, \ldots, j_{K}\right\}$ be the set of the first $K$ items in $S^{*}$. Let $S$ be the set the algorithm extends from the set $Y$. It suffices to prove the desired approximation result for $S$ since the greedy algorithm enumerates all sets of size $K$.

We may assume $S \neq S^{*}$. Let $S^{0} \subset S$ be the last set that the algorithm considers while extending $Y$ such that $S^{0} \subset$ $S^{*}$. Note that $Y \subseteq S^{0}$. From the assumption $S \neq S^{*}$, we know that $S^{0} \neq S$ because otherwise the greedy algorithm should not stop at $S$, and $S^{0} \neq S^{*}$ because otherwise $S$ is strictly better than $S^{*}$. Following the set $S^{0}$, let the items added into the solution by the greedy algorithm be $i_{1}, \ldots, i_{T}$. Let $i_{T+1} \in S^{*}$ be the first one excluded by the greedy algorithm because of the budget overflow. It is without loss of generality since if some item is neither in $S^{*}$ nor in the greedy solution, then we may remove it from the universe without affecting the analysis. Item $i_{T+1}$ must exist because $S \neq S^{*}$. Otherwise since every item is considered at some time during the greedy algorithm, it must be the case that $S^{*} \subset S$. Then $F(S)>F\left(S^{*}\right)$, which is a contradiction.

Let $S^{t}=S^{0} \cup\left\{i_{1}, \ldots i_{t}\right\}$. We call these sets partial solutions of the greedy algorithm. Let $c_{t}=\left(F\left(S^{t}\right)-\right.$ $\left.F\left(S^{t-1}\right)\right) / b_{i_{t}}$. Define $g\left(S^{t}\right)=F\left(S^{t}\right)-F\left(S^{0}\right)$, and let $g\left(S^{*}\right)=F\left(S^{*}\right)-F\left(S^{0}\right)$. Denote $\bar{B}=B-b\left(S^{0}\right)$. Note that the greedy solution $S$ may be strictly larger than $S^{T}$. But we will show $F\left(S^{T}\right)$ is large enough to give us the approximation ratio we need.

We will first show that when $\alpha^{\prime}=\alpha$, the partial solution $S^{T}$ obtained by extending $Y$ as defined earlier, achieves the desired approximation ratio $1-e^{-\alpha}$. In the end we will show the case when $\alpha^{\prime} \neq \alpha$.

We decompose $F\left(S^{T}\right)=F\left(S^{0}\right)+g\left(S^{T}\right)$ and replace $g\left(S^{T}\right)$ by two terms $g\left(S^{T}\right)-g\left(S^{T+1}\right)$ and $g\left(S^{T+1}\right)$. We then lower bound each term separately.

First we lower bound the value of $g\left(S^{T}\right)-g\left(S^{T+1}\right)$. The lower bound is a generalization of an inequality in [5]. For an item $j \in \mathcal{U} \backslash Y$, and set $Z \subseteq \mathcal{U} \backslash\left\{j_{1}, \ldots, j_{K}, j\right\}$, we can show the following upperbound of the difference between $F(Y \cup Z \cup\{j\})$ and $F(Y \cup Z)$

$$
F(Y \cup Z \cup\{j\})-F(Y \cup Z) \leq \frac{1}{K} F(Y)
$$

To see this, recall we order $S^{*}=\left\{j_{1}, \ldots, j_{\left|S^{*}\right|}\right\}$ where the element with smaller index has larger marginal increase, and $Y$ is the set of the first $K$ items among $S^{*}$. So the following inequalities hold for any $0 \leq t \leq K-1$.

$$
\begin{aligned}
& F(Y \cup Z \cup\{j\})-F(Y \cup Z) \\
\leq & F\left(\left\{j_{1}, \ldots, j_{t}\right\} \cup\{j\}\right)-F\left(\left\{j_{1}, \ldots, j_{t}\right\}\right) \\
\leq & F\left(\left\{j_{1}, \ldots, j_{t}, j_{t+1}\right\}\right)-F\left(\left\{j_{1}, \ldots, j_{t}\right\}\right)
\end{aligned}
$$

Summing up all inequalities of form $F(Y \cup Z \cup\{j\})-$ $F(Y \cup Z) \leq F\left(\left\{j_{1}, \ldots, j_{t}, j_{t+1}\right\}\right)-F\left(\left\{j_{1}, \ldots, j_{t}\right\}\right)$ for $0 \leq$ $t \leq K-1$ we have the desired inequality. Let $Y \cup Z=S^{T}$ and $j=i_{T+1}$, we then have $g\left(S^{T}\right)-g\left(S^{T+1}\right) \geq-\frac{1}{K} F(Y)$.

To bound $g\left(S^{T+1}\right)$, we will show it is a constant fraction of $g\left(S^{*}\right)$. First we use the definition of $\alpha$ and the property of the increasing submodular function (2) to upper bound $g\left(S^{*}\right)$ as follows.

$$
g\left(S^{*}\right) \leq \min _{1 \leq t \leq T}\left\{g\left(S^{t}\right)+\frac{1}{\alpha} \bar{B} c_{t+1}\right\}
$$

To prove this, first we show for any $S^{t}, 1 \leq t \leq T$, $F\left(S^{*} \cup S^{t}\right)-F\left(S^{t}\right) \geq \alpha\left(F\left(S^{*}\right)-F\left(S^{t}\right)\right)$. Let $i^{*}$ be an item that attains the maximum in (3). Since Algorithm 1 enumerates all sets of size $K$, there must be a set including the $i^{*}$ defined in $(3)$. Let $S^{1}$ be the first partial solution that includes $i^{*}$. Therefore we have $F\left(S^{t}\right) \geq F\left(i^{*}\right), F\left(S^{t}\right)<$ $F\left(S^{*}\right)$ and $F\left(S^{t} \cup S^{*}\right) \geq F\left(\left\{i^{*}\right\} \cup S^{*}\right)$ by the monotonicity of $F$. Also $F$ is nonnegative everywhere. Then

$$
\begin{aligned}
\frac{F\left(S^{*} \cup S^{t}\right)-F(S)}{F\left(S^{*}\right)-F\left(S^{t}\right)} & \geq \frac{F\left(S^{*} \cup S^{t}\right)-F\left(\left\{i^{*}\right\}\right)}{F\left(S^{*}\right)-F\left(\left\{i^{*}\right\}\right)} \\
& \geq \frac{F\left(S^{*} \cup\left\{i^{*}\right\}\right)-F\left(\left\{i^{*}\right\}\right)}{F\left(S^{*}\right)-F\left(\left\{i^{*}\right\}\right)} \\
& =\alpha .
\end{aligned}
$$

Therefore we have $F\left(S^{*}\right) \leq F\left(S^{t}\right)+$ $\frac{1}{\alpha}\left(F\left(S^{*} \cup S^{t}\right)-F\left(S^{t}\right)\right)$. Apply (2) and we have

$$
F\left(S^{*}\right) \leq F\left(S^{t}\right)+\frac{1}{\alpha} \sum_{i \in S^{*} \backslash S^{t}}\left(F\left(S^{t} \cup\{i\}\right)-F\left(S^{t}\right)\right)
$$


Replace $F$ by $g$, and we have

$$
\begin{aligned}
g\left(S^{*}\right) & \leq g\left(S^{t}\right)+\frac{1}{\alpha} \sum_{i \in S^{*} \backslash S^{t}}\left(g\left(S^{t} \cup\{i\}\right)-g\left(S^{t}\right)\right) \\
& \leq g\left(S^{t}\right)+\frac{1}{\alpha} \sum_{i \in S^{*} \backslash S^{t}} b_{i} c_{t+1} \\
& \leq g\left(S^{t}\right)+\frac{1}{\alpha} \sum_{i \in S^{*} \backslash S^{0}} b_{i} c_{t+1} \\
& =g\left(S^{t}\right)+\frac{1}{\alpha}\left(b\left(S^{*}\right)-b\left(S^{0}\right)\right) c_{t+1} \\
& \leq g\left(S^{t}\right)+\frac{1}{\alpha}\left(B-b\left(S^{0}\right)\right) c_{t+1} .
\end{aligned}
$$

We break down the right side of (5) into a summation of smaller increments. This is achievable because $g\left(S^{t+1}\right)-$ $g\left(S^{t}\right)=F\left(S^{t+1}\right)-F\left(S^{t}\right)=c_{t} b_{i_{t}}$ and $b_{i_{t}}$ is an integer. In particular, let $\bar{B}_{t}=b\left(S^{t}\right)-b\left(S^{0}\right), 1 \leq t \leq T+1$, and $\bar{B}_{0}=0$. Also recall $\bar{B}=B-b\left(S^{0}\right)$. Define $\rho_{l}, 1 \leq l \leq$ $\bar{B}_{T+1}$ as follows: $\rho_{l}=c_{t}$ if $\bar{B}_{t-1}+1 \leq l \leq \bar{B}_{t}$. Therefore we have $g\left(S^{t}\right)=\sum_{l=1}^{\bar{B}_{t}} \rho_{l}$. Notice that $\rho_{1}, \ldots, \rho_{\bar{B}_{T+1}}$ is a nonincreasing sequence. Then the right side of (5) can be written in the following way.

$$
\min _{1 \leq t \leq T}\left\{g\left(S^{t}\right)+\frac{\bar{B}}{\alpha} c_{t+1}\right\}=\min _{1 \leq s \leq B_{T+1}}\left\{\sum_{j=1}^{s-1} \rho_{j}+\frac{\bar{B}}{\alpha} \rho_{s}\right\} .
$$

To see this, we can rewrite the left side of $(6)$ in the following way.

$$
\min _{1 \leq t \leq T}\left\{g\left(S^{t}\right)+\frac{\bar{B}}{\alpha} c_{t+1}\right\}=\min _{1 \leq t \leq T}\left\{\sum_{l=1}^{B_{t}} \rho_{l}+\frac{\bar{B}}{\alpha} \rho_{B_{t+1}}\right\} .
$$

Then we claim that

$$
\min _{1 \leq t \leq T}\left\{\sum_{l=1}^{B_{t}} \rho_{l}+\frac{\bar{B}}{\alpha} \rho_{B_{t+1}}\right\}=\min _{1 \leq s \leq B_{T+1}}\left\{\sum_{j=1}^{s-1} \rho_{j}+\frac{\bar{B}}{\alpha} \rho_{s}\right\} .
$$

Assume that $\min _{1 \leq s \leq \bar{B}_{T+1}}\left\{\sum_{l=1}^{s-1} \rho_{l}+\frac{\bar{B}}{\alpha} \rho_{s}\right\}$ does not attain minimum at an $s=\bar{B}_{t}+1$ for some $t$. Let $\bar{B}_{t}+1<s \leq$ $\bar{B}_{t+1}$ for some $t$. Then $\sum_{l=1}^{\bar{B}_{t}} \rho_{l}+\frac{\bar{B}}{\alpha} \rho_{\bar{B}_{t}+1}<\sum_{l=1}^{s-1} \rho_{l}+\frac{\bar{B}}{\alpha} \rho_{s}$ because $\bar{B}_{t}<s-1$ and $\rho_{\bar{B}_{t}+1} \leq \rho_{s}$. This leads to a contradiction.

To bound the right side of (6), we need the following inequality. For a positive integer $P, D>0, \rho_{l} \geq 0$ for $1 \leq$ $l \leq P$, and $\rho_{1}>0$, then

$$
\frac{\sum_{l=1}^{P} \rho_{l}}{\min _{t=1, \ldots, P}\left(\sum_{l=1}^{t-1} \rho_{l}+D \rho_{t}\right)} \geq 1-\left(1-\frac{1}{D}\right)^{P} \geq 1-e^{-\frac{P}{D}} .
$$

The above inequality is analogous to one in [18, where the only difference is that $D$ is a positive integer. However $D$ is not required to be an integer for the validity of the inequality. Notice $g\left(S^{T+1}\right)=\sum_{l=1}^{\bar{B}_{T+1}} \rho_{l}$. Combine (5) and (6) and apply (7) by setting $P=\bar{B}_{T+1}, D=\bar{B} / \alpha$, we have

$$
\frac{g\left(S^{T+1}\right)}{g\left(S^{*}\right)} \geq \frac{\sum_{l=1}^{\bar{B}_{T+1}} \rho_{l}}{\min _{1 \leq s \leq \bar{B}_{T+1}}\left(\sum_{j=1}^{s-1} \rho_{j}+\frac{\bar{B}}{\alpha} \rho_{s}\right)} \geq 1-e^{-\alpha \bar{B}_{T+1} / \bar{B}} .
$$

Since adding item $i_{T+1}$ makes the budget overflow, we have $b\left(S^{T}\right)+b_{T+1}>B$. Therefore $\bar{B}_{T+1}=b\left(S^{T}\right)+$ $b_{T+1}-b\left(S^{0}\right) \geq B-b\left(S^{0}\right)=\bar{B}$, and $g\left(S^{T+1}\right) \geq$ $\left(1-e^{-\alpha \bar{B}_{T+1} / \bar{B}}\right) g\left(S^{*}\right) \geq\left(1-e^{-\alpha}\right) g\left(S^{*}\right)$. Note that we have used the fact $\bar{B}_{T+1} \geq \bar{B}$, where the inequality is actually strict. This is the same as in the proof of $(1-1 / e)$ in 5 .

Therefore we have

$$
\begin{aligned}
F(S) & \geq F\left(S^{T}\right)=F\left(S^{0}\right)+g\left(S^{T}\right)-g\left(S^{T+1}\right)+g\left(S^{T+1}\right) \\
& \geq F\left(S^{0}\right)-\frac{1}{K} F(Y)+\left(1-e^{-\alpha}\right) g\left(S^{*}\right) \\
& =F\left(S^{0}\right)-F(Y)+F(Y)-\frac{1}{K} F(Y)+\left(1-e^{-\alpha}\right) g\left(S^{*}\right) .
\end{aligned}
$$

Lower bound $F\left(S^{0}\right)-F(Y)$ by $\left(1-e^{-\alpha}\right)\left(F\left(S^{0}\right)-F(Y)\right)$ and $F(Y)-\frac{1}{K} F(Y)$ by $\left(1-e^{-\alpha}\right) F(Y)$, we have the inequality above is at least $\left(1-e^{-\alpha}\right)\left(F\left(S^{0}\right)+\left(g\left(S^{*}\right)\right)=\right.$ $\left(1-e^{-\alpha}\right) F\left(S^{*}\right)$

Now notice for $\alpha^{\prime}<\alpha$, as long as $K=\left\lceil e^{\alpha^{\prime}}\right\rceil$, The inequality above can be lower bounded by $\left(1-e^{-\alpha^{\prime}}\right) F\left(S^{*}\right)$. For $\alpha^{\prime}>\alpha$, since we enumerate more subsets $\left(K>e^{\alpha}\right)$, the approximation ratio $1-e^{-\alpha}$ can also be achieved.

\subsection{Combining SAA and Algorithm 1}

Since $\mathrm{SP}$ and $\mathrm{SA}$ are special cases of $\mathrm{SA} 0$ and (SP0), and we know that (SA) is a submodular maximization problem, we have the following result.

Theorem 8. Assume that $\max _{i} \operatorname{Var}\left[\tilde{a}_{i}\right]$ can be bounded by a constant. Given $\gamma>0$ and $\alpha^{\prime} \geq 1$, with probability at least $1-2 \gamma$, Algorithm 1 solves the stochastic problem (SP) with approximation ratio $\left(1-e^{-\min \left(\alpha, \alpha^{\prime}\right)}\right)(1-\epsilon)-\epsilon$ in time poly $\left(2^{e^{\alpha^{\prime}}}, n, \frac{1}{\epsilon}, \log \frac{1}{\gamma}\right)$.

Proof. Recall $\sigma^{2}=\max _{S \subseteq \mathcal{U}}\{\operatorname{Var}[f(\tilde{a}(S))] \mid b(S) \leq B\}$. By Theorem 3. we need $\frac{2 \sigma^{2}}{\epsilon^{2}} \log \frac{|X|}{\gamma}$ i.i.d. samples of $\tilde{a}$ to ensure that the sample average approximation is close to the expected value with probability at least $1-2 \gamma$. Take the upper bound of $|X|$ as $2^{n}$ then $N=\frac{2 \sigma^{2}}{\epsilon^{2}}(n+$ $\left.\log \frac{1}{\gamma}\right)$ in SA. Since $f$ is a concave function, we have $f(a(S)) \leq f(0)+f^{\prime}(0) \cdot a(\mathcal{U})$ for every $S$ and every sample $a$. Therefore $\operatorname{Var}[f(\tilde{a}(S))] \leq\left(f^{\prime}(0)\right)^{2} \cdot \operatorname{Var}[\tilde{a}(\mathcal{U})] \leq$ $\left(f^{\prime}(0)\right)^{2} \cdot\left(n \max _{i} \operatorname{Var}\left[\tilde{a}_{i}\right]+n^{2} \max _{i, j} \operatorname{Cov}\left[\tilde{a}_{i}, \tilde{a}_{j}\right]\right)$. Notice that $\operatorname{Cov}\left[\tilde{a}_{i}, \tilde{a}_{j}\right] \leq \sqrt{\operatorname{Var}\left[\tilde{a}_{i}\right]} \sqrt{\operatorname{Var}\left[\tilde{a}_{j}\right]}$, therefore $\sigma^{2}=$ $O\left(n^{2}\right)$ by our assumption that $\max _{i} \operatorname{Var}\left[\tilde{a}_{i}\right]$ is upper bounded by a constant. We enumerate all sets of size $K \geq$ $e^{\alpha^{\prime}}$ in Algorithm 1 and for each set of size $K$, extending it 
greedily needs time polynomial in $n$ and $N$. Therefore the total running time is poly $\left(2^{e^{\alpha^{\prime}}}, n, \frac{1}{\epsilon}, \log \frac{1}{\gamma}\right)$. Combining Theorem 6 and Theorem 3 the approximation ratio follows.

\subsection{Power utility function}

In this section, we consider a particular class of concave utility function: power utility function. Let the concave utility function in $\mathrm{SP}$ be $f(t)=t^{p}, 0<p<1$. It is also called isoelastic function for utility or constant relative risk aversion (CRRA) utility function in the economics literature. We show that we can calculate an approximation ratio that only depends on the exponent $p$ and the budget $B$.

For power utility function $f=t^{p}$, the following problem is a special case of $\mathrm{SA} 0$.

$$
\max _{S \subseteq \mathcal{U}}\left\{F(S)=\sum_{r=1}^{N} f\left(a_{r}(S)\right) \mid b(S) \leq B\right\},
$$

where $a_{r}=\left(a_{r 1}, \ldots, a_{r n}\right) \in \mathbb{R}_{+}^{n}$. Let $S^{*}$ be an optimal solution in the following.

Recall that set $S^{1}$ is the first partial solution constructed by the greedy algorithm that is not contained in $S^{*}$, ie., $S^{1} \backslash S^{*} \neq \emptyset$. We first give a lower bound of the ratio between $F\left(S^{*} \cup S^{1}\right)-F\left(S^{1}\right)$ and $F\left(S^{*}\right)-F\left(S^{1}\right)$ in terms of $p$ and $B$. Then for power utility functions, we can write the approximation ratio of the greedy algorithm as a function of $p$ and $B$. Then we upper bound $K$, which is the size of enumeration in Algorithm 1, by a function only depending on $p$ so that Algorithm 1 runs in polynomial time when $p$ is a constant. We assume that $B>1$.

Lemma 9. Given a problem (SAA-P) with utility function $f(t)=t^{p}$ and a knapsack constraint $B, F\left(S^{*} \cup\right.$ $\left.S^{1}\right)-F\left(S^{1}\right) \geq \alpha(B, p)\left(F\left(S^{*}\right)-F\left(S^{1}\right)\right)$, where the function $\alpha(B, p)=\frac{\left(B^{1 / p}+1\right)^{p}-1}{B-1}$ which is decreasing in $B$ when $B>1$.

Proof. Let $S^{0}$ be the partial solution just before $S^{1}$. First we lower bound the difference between $F\left(S^{1}\right)$ and $F\left(S^{0}\right)$ :

$$
F\left(S^{1}\right)-F\left(S^{0}\right) \geq \frac{1}{B}\left(F\left(S^{*}\right)-F\left(S^{0}\right)\right) .
$$

Let $c_{1}=\frac{F\left(S^{1}\right)-F\left(S^{0}\right)}{b_{i_{1}}}$. By (2), we have $F\left(S^{*}\right)-F\left(S^{0}\right) \leq \sum_{j \in S^{*} \backslash S^{0}} F\left(S^{0} \cup\{j\}\right)-F\left(S^{0}\right)=$ $\sum_{j \in S^{*} \backslash S^{0}} b_{j} \frac{F\left(S^{0} \cup\{j\}\right)-F\left(S^{0}\right)}{b_{j}}$. By the greedy algorithm, $c_{1} \geq \max \left\{\frac{F\left(S^{0} \cup\{j\}\right)-F\left(S^{0}\right)}{b_{j}} \mid j \in S^{*} \backslash S^{0}\right\}$, therefore $F\left(S^{*}\right)-F\left(S^{0}\right) \leq c_{1} \sum_{j \in S^{*} \backslash S^{0}} b_{j} \leq c_{1} B$. Replace $c_{1}$ by $\left(F\left(S^{1}\right)-F\left(S^{0}\right)\right) / b_{i_{1}}$, we have $F\left(S^{1}\right)-F\left(S^{0}\right) \geq$ $\frac{b_{i_{1}}}{B}\left(F\left(S^{*}\right)-F\left(S^{0}\right)\right) \geq \frac{1}{B}\left(F\left(S^{*}\right)-F\left(S^{0}\right)\right)$, where the last inequality comes from the fact that $b_{i} \in \mathbb{N}_{+}$.

For $1 \leq r \leq N$, let $x_{r}=a_{r}\left(S^{1} \backslash S^{*}\right), y_{r}=a_{r}\left(S^{1} \cap\right.$ $\left.S^{*}\right)=a_{r}\left(S^{0}\right)$ and $A_{r}=a_{r}\left(S^{*}\right)$ so that $\left(A_{r}+x_{r}\right)^{p}=$
$f\left(a_{r}\left(S^{*} \cup S^{1}\right),\left(x_{r}+y_{r}\right)^{p}=f\left(a_{r}\left(S_{1}\right)\right), A_{r}^{p}=f\left(a_{r}\left(S^{*}\right)\right)\right.$. Let $x=\left(x_{1}, \ldots, x_{N}\right), y=\left(y_{1}, \ldots, y_{N}\right)$. Define

$$
h(x, y)=\frac{\sum_{r=1}^{N}\left(A_{r}+x_{r}\right)^{p}-\sum_{r=1}^{N}\left(x_{r}+y_{r}\right)^{p}}{\sum_{r=1}^{N} A_{r}^{p}-\sum_{r=1}^{N}\left(x_{r}+y_{r}\right)^{p}},(x, y) \in X
$$

where

$$
X=\left\{(x, y) \mid \sum_{r=1}^{N}\left(x_{r}+y_{r}\right)^{p}-\sum_{r=1}^{N} y_{r}^{p} \geq \frac{1}{B}\left(\sum_{r=1}^{N} A_{r}^{p}-\sum_{r=1}^{N} y_{r}^{p}\right)\right\}
$$

Our goal is to give a lower bound of the ratio $\frac{F\left(S^{*} \cup S^{1}\right)-F\left(S^{1}\right)}{F\left(S^{*}\right)-F\left(S^{1}\right)}$. By $[9]$, we know that the ratio is at least $\min \{h(x, y) \mid(x, y) \in X\}$. A careful calculation using Lagrange multiplier can show that

$$
\min \{h(x, y) \mid(x, y) \in X\}=\frac{\left(B^{1 / p}+1\right)^{p}-1}{B-1} .
$$

By calculating its derivative, we may also prove that the function $\alpha(B, p)$ is decreasing in $B$ when $B>1$.

Theorem 10. For the power utility function $f(t)=t^{p}$ where $p$ is a constant, given $\gamma>0$, with probability at least $1-2 \gamma$, Algorithm 1 runs in time poly $\left(n, \frac{1}{\epsilon}, \log \frac{1}{\gamma}\right)$ and solves the stochastic problem $\sqrt{\mathrm{SP}}$ with approximation ratio $\left(1-e^{-\alpha(B, p)}\right)(1-\epsilon)-\epsilon$.

Proof. By Lemma 9, we know that for $B>1$ and integral, $\alpha(B, p)$ is at most $\alpha(2, p)$. Set $K=\left\lceil e^{\alpha(2, p)}\right\rceil$ and the algorithm runs in time poly $\left(n, \frac{1}{\epsilon}, \log \frac{1}{\gamma}\right)$ by Corollary 8 By a close examination of the proof of inequality (5), we know that $\alpha$ can be replaced by the ratio $\frac{F\left(S^{*} \cup S^{1}\right)-F\left(S^{1}\right)}{F\left(S^{*}\right)-F\left(S^{1}\right)}$. By Lemma 9, the ratio can be lower bounded by $\alpha(B, p)$. Thus the approximation ratio of Algorithm 1 on (SAA-P is $1-e^{-\alpha(B, p)}$ by Theorem 6 . The approximation of the stochastic problem $\mathrm{SP}$ then follows from Corollary 8

\section{Acknowledgement}

This research has been supported in part by the National Science Foundation (Grant 1129871) and the Air Force Office of Scientific Research (Grant FA9550-12-1-0154).

\section{References}

[1] J. Von Neumann, O. Morgenstern, Theory of games and economic behavior, Princeton University Press, 1944.

[2] P. J. Schoemaker, The expected utility model: Its variants, purposes, evidence and limitations, Journal of Economic Literature 20 (2) (1982) 529-563.

[3] S. Ahmed, A. Atamtürk, Maximizing a class of submodular utility functions, Mathematical Programming 128 (1) (2011) 149169.

[4] U. Feige, A threshold of $\ln n$ for approximating set cover, Journal of the ACM 45 (4) (1998) 634-652.

[5] M. Sviridenko, A note on maximizing a submodular set function subject to a knapsack constraint, Operations Research Letter 32 (1) (2004) 41-43. 
[6] A. Shapiro, Monte carlo sampling methods, in: A. Ruszczyński, A. Shapiro (Eds.), Stochastic Programming, Vol. 10 of Handbooks in Operations Research and Management Science, Elsevier, 2003, pp. $353-425$.

[7] C. Swamy, D. B. Shmoys, Sampling-based approximation algorithms for multi-stage stochastic, in: Proceedings of 46th Annual IEEE Symposium on Foundations of Computer Science, FOCS, 2005, pp. 357-366.

[8] M. Charikar, C. Chekuri, M. Pál, Sampling bounds for stochastic optimization, in: Proceedings of the 9th International Workshop on Randomization and Computation, RANDOM, 2005, pp. 257-269.

[9] J. Vondràk, Submodularity in combinatorial optimization, Ph.D. thesis, Charles University, Prague (2007).

[10] M. Conforti, G. Cornuéjols, Submodular set functions, matroids and the greedy algorithm: Tight worst-case bounds and some generalizations of the Rado-Edmonds theorem, Discrete Applied Mathematics 7 (3) (1984) 251-274.

[11] J. Vondràk, Submodularity and curvature: the optimal algorithm, RIMS Kokyuroku Bessatsu B23, Kyoto (2010) 253-266.

[12] J. Li, A. Deshpande, Maximizing expected utility for stochastic combinatorial optimization problems, in: Proceedings of the 52nd Annual IEEE Symposium on Foundations of Computer Science, FOCS, 2011, pp. $797-806$.

[13] T. Klastorin, On a discrete nonlinear and nonseparable knapsack problem, Operations Research Letters 9 (4) (1990) 233237.

[14] A. Asadpour, H. Nazerzadeh, A. Saberi, Stochastic submodular maximization, in: Proceedings of the 4th International Workshop on Internet and Network Economics, WINE, 2008, pp. 477-489.

[15] A. Mehrez, Z. Sinuany-Stern, Resource allocation to interrelated risky projects using a multiattribute utility function, Management Science 29 (4) (1983) 430-439.

[16] B. C. Dean, M. X. Goemans, J. Vondrk, Approximating the stochastic knapsack problem: The benefit of adaptivity, Mathematics of Operations Research 33 (4) (2008) 945-964. doi: $10.1287 /$ moor. 1080.0330

[17] A. Shapiro, D. Dentcheva, A. Ruszczyński, Lectures on Stochastic Programming: Modeling and Theory, Society for Industrial and Applied Mathematics, 2009.

[18] L. A. Wolsey, Maximising real-valued submodular functions: Primal and dual heuristics for location problems, Mathematics of Operations Research 7 (3) (1982) 410-425. 